\title{
Development of single-tube nested real-time PCR assays with long internally quenched probes for detection of norovirus genogroup II
}

\author{
Hongyan Xia ${ }^{1,2}$, Sabine Gravelsina ${ }^{1,3}$, Christina Öhrmalm¹, Jakob Ottoson ${ }^{2,4}$, Jonas Blomberg ${ }^{1}$ \\ ${ }^{1}$ Section of Clinical Virology, Department of Medical Sciences, Uppsala University, Sweden, ${ }^{2}$ Swedish University \\ of Agricultural Sciences, Department of Biomedical Sciences and Veterinary Public Health, Uppsala, Sweden, \\ ${ }^{3}$ August Kirchenstein Institute of Microbiology and Virology, Riga Stradins University, Riga, Latvia, and ${ }^{4}$ National \\ Food Administration, Department of Risk and Benefit Assessment, Uppsala, Sweden
}

BioTechniques 60:28-34 (January 2016) doi 10.2144/000114370
Keywords: real-time PCR; variation-tolerant capture multiplex assay; norovirus

The high sequence variation of RNA viruses necessitates use of degenerate primers and probes or multiple primers and probes in molecular diagnostic assays. We showed previously that PCR amplification in two rounds, first with long target-specific primers and then with short generic primers, followed by detection using long probes, can tolerate sequence variation. Here we demonstrate that long primers and probes of up to 56 nucleotides can also be applied in real-time PCR for the detection of norovirus genogroup II with improved sensitivity. Probe design (method of incorporating quenchers, use of Zen internal quencher or traditional quenchers) greatly affects the sensitivity of the real-time PCR assays.

Norovirus is a major cause of human gastroenteritis and has been identified as the predominant cause of food-borne outbreaks (1). Both food and water are important sources of norovirus transmission. Rapid and accurate detection is important for diagnosis of norovirus in patients and for tracing the source of the virus in food and environmental samples. Due to its high sensitivity and specificity, real-time PCR has become the gold standard for detection of norovirus from different sources $(2,3)$. Most real-time PCR assays for noroviruses target the conserved region in the junction between ORF1 and ORF2 (4-7). However, due to high sequence variation, a high degree of sequence diversity is observed even in the conserved region. To improve the detection rate and to accommodate norovirus divergence, degenerate primers and multiple primers and probes have been used for the detection of different genogroups in real-time PCR assays $(4,8)$. Modified oligonucleotides have also been used to compensate for norovirus sequence polymorphisms (9). However, all of these assays use very short primers and probes and are not highly variation-tolerant. Considering the characteristic sequence diversity of norovirus, an assay that can tolerate sequence variation may greatly reduce the false negative rate.

We showed previously that long primers and probes can tolerate sequence variation for norovirus genogroup II (GII) and rotavirus in a variation-tolerant capture multiplex assay (VOCMA) with hybridization readout (10). In the first round of PCR amplification, VOCMA uses a low concentration of long target-specific primers designed to allow hybridization despite target variation, and this is followed by a second amplification round using short generic primers. In the previous version of VOCMA, we detected the PCR products using probes in a suspension microarray in a Luminex multianalyzer after hybridization. This system allowed for the use of highly multiplexed panels for the screening of clinical samples. In that previous work, sensitive and specific 22-plex sepsis and 7-plex gastro VOCMA panels were created, but problems with this system included the additional labor needed for the readout and the potential for contamination when the PCR tubes were opened before the hybridization step.

Here we demonstrate that the VOCMA principle can be applied to real-time PCR conditions using nuclease-activated (TaqMan) internally quenched probes. In comparison with detection of the PCR product in a Luminex machine, real-time PCR, which combines amplification with detection, shortens both handling and running times. Single-tube nested real-time PCR (11) has been shown to have especially high sensitivity for detection of pathogens such as Mycobacterium tuberculosis, retroviruses, and hepatitis A virus (11-13). The risk of cross-contamination is low since the sequential reactions occur in a single tube. It is therefore reasonable to adapt VOCMA for use with single-

\section{METHOD SUMMARY}

The variation-tolerant capture multiplex assay (VOCMA) requires long degenerate primers and probes for the initial round of PCR amplification. We developed a norovirus genogroup II VOCMA with nuclease-activated probes where internal quenchers were used. 


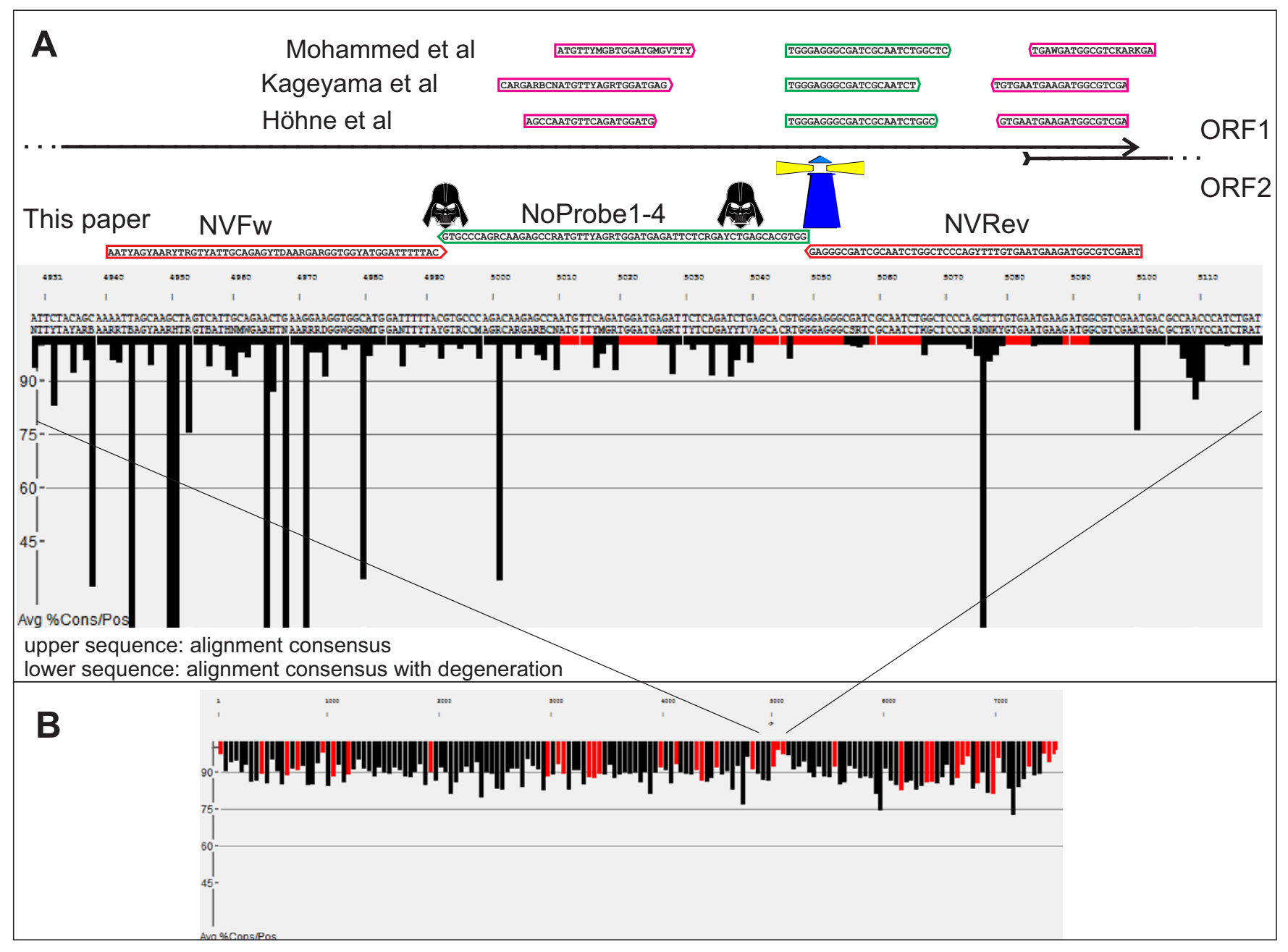

Figure 1. Positions and sequences of primers and probes used for real-time PCR assays targeting the conserved region in the junction between ORF1 and ORF2 of norovirus genogroup II. (A) Top: Primers and probes used for three previously published norovirus Gll real-time PCR assays targeting the conserved ORF1-ORF2 overlap region. Bottom: Sequences and positions of the primers and probes (all tolerant of norovirus Gll variation) used in our real-time PCR assay targeting the conserved ORF1-ORF2 overlap region, as shown by the Consort program (J.B., unpublished manuscript). The sequence of the conserved ORF1-ORF2 overlap region shown is the consensus derived from the BLAST alignment of 584 full-length norovirus genogroup II sequences using the Lordsdale sequence as the query sequence. The red regions in sequence alignment depict positions without variation; $y$-axis: average $\%$ conservation per position; $\mathrm{x}$-axis: alignment position. For the primer sequence, the approximate positions of quenchers (black symbol) and fluorophore (lighthouse symbol) are shown. Note that NoProbes 1-4 are antisense. (B) The entire alignment of the 584 full-length norovirus genogroup II sequences.

tube nested real-time PCR. However, in order to maintain the variation tolerance, the specific probes in VOCMA are often 50-70 nucleotides, which makes them unsuitable as conventional TaqMan end-labeled probes for real-time PCR. Normally, efficient quenching requires that the quencher be situated less than 30 bases from the fluorophore. In order to maintain quenching efficiency for the long probes used in VOCMA, quenchers must be placed internally in the probe. Black Hole Quenchers (BHQs) have been widely used in real-time PCR due to their improved quenching compared with other quenchers and their ability to be used with a wider range of reporter dyes, expanding the options available for multiplexed or genotyping assays. The $\mathrm{BHQ}$ can be placed internally by BHQ DMT amidite or $\mathrm{BHQ}$ dT linker amidite chemistry, but since these options have rarely been used and may affect the function of the probes, we have now evaluated their use in synthesizing VOCMA probes. Furthermore, we also tested the recently developed ZEN internal quencher from Integrated DNA Technologies (IDT), which can decrease the distance between the dye and quencher to only nine nucleotides and significantly reduces background fluorescence, resulting in improved sensitivity.

Human noroviruses consist of the GI, Gll or GIV genogroups. The Gll genogroup is the most prevalent, and it carries much of the variation in human norovirus. Therefore, in the present study, Gll was used to evaluate several TaqMan probes in a real-time PCR based on the VOCMA principle. A synthetic oligonucleotide from the highly conserved ORF1ORF2 junction region of Gll was used as target for PCR amplification. Four TaqMan probes with the same nucleotide sequence but different quencher combinations were tested in the real-time PCR assays, and the sensitivity of the assays was compared.

\section{Materials and methods}

\section{Design of primers and probes}

Whole genome sequences of Gll noroviruses $(n=580)$ collected in BLASTN ( $\mathrm{NCBI}, \mathrm{NIH}$, Bethesda, MA) were analyzed to map nucleotide conservation and variation using the Consort program 
Table 1: Primers, probes, and synthetic oligonucleotides for norovirus genogroup II

\begin{tabular}{|c|c|c|}
\hline Primers/Probes & Nucleotide sequences $\left(5^{\prime}-3^{\prime}\right)$ & Location \\
\hline Generic first & TTGGATAAGTGGGATA & \\
\hline Generic second & AAGATATCGTAAGGAT & \\
\hline NVFw & AAGATATCGTAAGGATAATYAGYAARYTRGTYATTGCAGAGYTDAARGARGGTGGYATGGATTTTTAC & $4942-4993$ \\
\hline NVRev & TTGGATAAGTGGGATAAYTCGACGCCATCTTCATTCACAAARCTGGGAGCCAGATTGCGATCGCCCTC & $5051-5102$ \\
\hline Noprobe1 & FAM/CCACGTGCTCAGRTCYGAGA/iBHQ1/ATCTCATCCAYCTRAACATYGGCTCTTGYCTGGGCAC/3Spacer C3 & $4994-5050$ \\
\hline Noprobe2 & FAM/CCACGTGCTCAGRTCYGAGAA[T(BHQ-1)]CTCATCCAYCTRAACATYGGCTCTTGYCTGGGCAC/spacer C3 & $4994-5050$ \\
\hline Noprobe3 & FAM/CCACGTGCT/zen/CAGRTCYGAGAATCTCATCCAYCTRAACATYGGCTCTTGYCTGGGCAC/3lowa black RQ-Sp & 4994-5050 \\
\hline Noprobe4 & FAM/CCACGTGCT/zen/CAGRTCYGAGAATCTCATCC/zen/AYCTRAACATYGGCTCTTGYCTGGGCAC/3lowa black RQ-Sp & $4994-5050$ \\
\hline Synthetic target & $\begin{array}{l}\text { AATTAGCAAGCTAGTCATTGCAGAACTGAAGGAAGGTGGCATGGATTTTTACGTGCCCAGACAAGAGCCAATGTTCAGATG- } \\
\text { GATGAGATTCTCAGATCTGAGCACGTGGGAGGGCGATCGCAATCTGGCTCCCAGCTTTGTGAATGAAGATGGCGTCGAAT }\end{array}$ & $4942-5102$ \\
\hline
\end{tabular}

(J. Blomberg, unpublished manuscript), which visualizes the frequency of variation and the variation in nucleotide composition at each base position as well as the number of aligned sequences (Figure 1). The design process, briefly described above, was based on the conserved ORF1-ORF2 overlap area (nucleotides
5085-5104 of the Lordsdale norovirus reference genome $\times 86557$ and its flanking sequence). The target for the forward primer (nucleotides 4942-4993) was less conserved, however. We addressed this issue by grouping the variation in this sequence into 5 major haplogroups differing by a maximum of 5 mismatches within the haplogroup and 10 mismatches between the haplogroup consensuses. This resulted in five suggested degenerate primer sequences. These were further evaluated against the entire variation of the alignment using the NucZip (14) and nearest neighbor algorithms (15) for prediction of hybridization. The chosen sequence was predicted to hybridize with all variant sequences. All of these sequence analysis functions are featured in the Consort program. The same procedure was used for construction of the probe and reverse primer. Finally, all oligonucleotides for the VOCMA amplification mixture were tested for interaction using the program Visual OMP (DNA Software, Ann Arbor, $\mathrm{MI})$ to avoid hairpin, homodimer, and heterodimer structures. We had to go through several rounds of optimization before finalizing the design of the primers. The long primers and probes containing degenerate positions were thus designed to allow variation tolerance (Table 1), a feature of VOCMA. The sequences of the generic primers from our previous study were added to the $5^{\prime}$ end of the primers to allow generic amplification of the specific target in the second round of PCR. The primers were synthesized by Integrated DNA Technologies (IDT, Coralville, IA). With a length of 56 nucleotides, the probe must be internally labeled with a quencher. All probes had the same nucleotide sequence and were labeled with FAM at the 5' end (Figure 2). Noprobe1 and Noprobe2 contained one internal $\mathrm{BHQ}$ quencher at positions 20 and 21, respectively, from the 5 end. The internal quencher was introduced between the $5^{\prime}$ and 3 ' deoxyribose positions in the phosphate-deoxyribose chain by $\mathrm{BHQ}$ DMT amidite chemistry for Noprobe1 and

(c) 2015 State Bar of Wisconsin. PINNACLE is a registered trademark of the State Bar of Wisconsin. All Rights Reserved. 


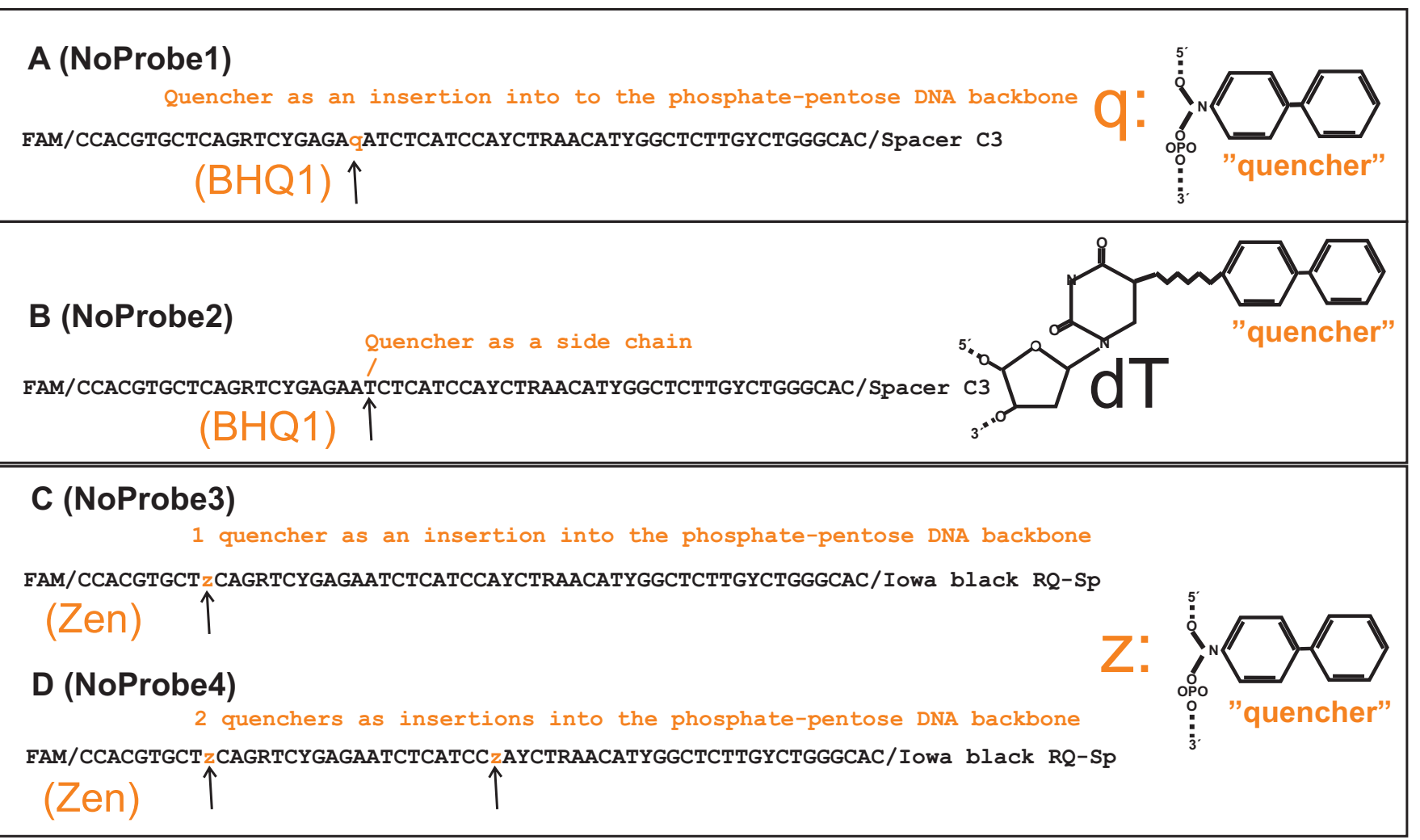

Figure 2. Structure of the four norovirus genogroup II probes. Regardless of the actual quencher chemistry, the quencher is symbolized by a biphenyl group. (A) Noprobe1 contains an internal BHQ1 quencher inserted into the phosphate-pentose backbone between nucleotides 20 and 21 (counting from the $5^{\prime}$ end). (B) Noprobe2 contains an internal BHQ1 quencher added as a side chain to the thymidine at position 22. (C) NoProbe3 contains an internal Zen quencher inserted between nucleotides 9 and 10 and an lowa Black quencher at the 3' end. (D) NoProbe4 is the same as NoProbe3, except with an additional Zen quencher inserted between nucleotides 29 and 30.

as a modified nucleotide by BHQ dT linker (LGC Biosearch Technologies; Petaluma, CA) amidite chemistry for Noprobe2. Noprobe3 had a single Zen quencher nine nucleotides from the 5 ' end. NoProbe4 had 2 Zen quenchers 9 and 29 nucleotides from the 5 ' end. Like NoProbe1, the Zen internal quencher was introduced into the phosphate-deoxyribose chains of NoProbe3 and NoProbe4. In addition to the Zen internal quenchers, Noprobe3 and Noprobe4 had an lowa Black quencher at their 3 ends. More information on internal Zen quenchers can be found at https://eu.idtdna.com/ pages/decoded/decoded-articles/coreconcepts/decoded/2013/03/29/modification-highlight-zen-internal-quencher.

\section{Synthetic target}

The oligonucleotide corresponding to nucleotides 4942-5102 of the Lordsdale strain (X86557) was synthesized by biomers.net (UIm, Germany) and used as the target for optimization of the real-time PCR conditions and determination of the sensitivity of the assay. The synthetic single-stranded DNA was dissolved in nuclease-free water and was serially diluted 10 -fold from $1 \times 10^{6}$ to $1 \times 10^{0}$ molecules/ $\mu \mathrm{L}$ with $20 \mathrm{ng} / \mu \mathrm{L}$ yeast RNA (Ambion, Austin, TX).

\section{Samples}

Feces samples obtained from hospital patients between October 2014 and February 2015 were provided anonymously with patient consent, according to the Swedish Biobank law and Uppsala University hospital rules, and extracted using the EasyMag system (Bio-Merieux, Marcy l'Etoile, France) according to the manufacturer's instructions.

A total of 86 clinical patient samples were used in this study. Of these samples, 46 were norovirus genogroup II, 23 were genogroup I, and 20 tested negative for genogroups I and II, as determined by routine diagnostics at the Clinical Microbiology Laboratory of Uppsala University Hospital based on a specific norovirus genogroup I and II real-time PCR assay (16).

Single-tube nested real-time PCR assay Single-tube nested real-time PCR was performed using a CFX 96 touch real-time PCR detection system (Bio-Rad, Hercules, CA) with the Path-ID Multiplex One-Step RT-PCR Kit (ThermoFisher Scientific, Foster City, CA) in a $25 \mu \mathrm{L}$ volume comprising $2 \mu \mathrm{L}$ extracted RNA or synthetic target and $23 \mu \mathrm{L}$ master mix containing: $1 \mu \mathrm{L} 25 \times$ multiplex RT-PCR enzyme mix, $12.5 \mu \mathrm{L} 2 \times$ multiplex RT-PCR buffer, 40 nM norovirus Gll specific forward (NvFw) and reverse (NVRev) primers, 300 $\mathrm{nM}$ generic primers, and $100 \mathrm{nM}$ probe. The specific and generic primers were included in the same master mix instead of being added separately in different PCR steps. In this way, cross-contamination was avoided. The real-time PCR conditions, essentially the same as previously developed for VOCMA (10), were: $45^{\circ} \mathrm{C}$ for $10 \mathrm{~min}$ for reverse transcription and $95^{\circ} \mathrm{C}$ for $10 \mathrm{~min}$, followed by nested real-time PCR. The first PCR was performed with 15 cycles at $95^{\circ} \mathrm{C}$ for $15 \mathrm{~s}$ for denaturation and $61^{\circ} \mathrm{C}$ for $45 \mathrm{~s}$ for annealing and extension. The second PCR was carried out with 40 cycles at $95^{\circ} \mathrm{C}$ for $15 \mathrm{~s}$ and $52^{\circ} \mathrm{C}$ for $45 \mathrm{~s}$. Nuclease-free water was used as a negative control and synthetic target as a positive control. 

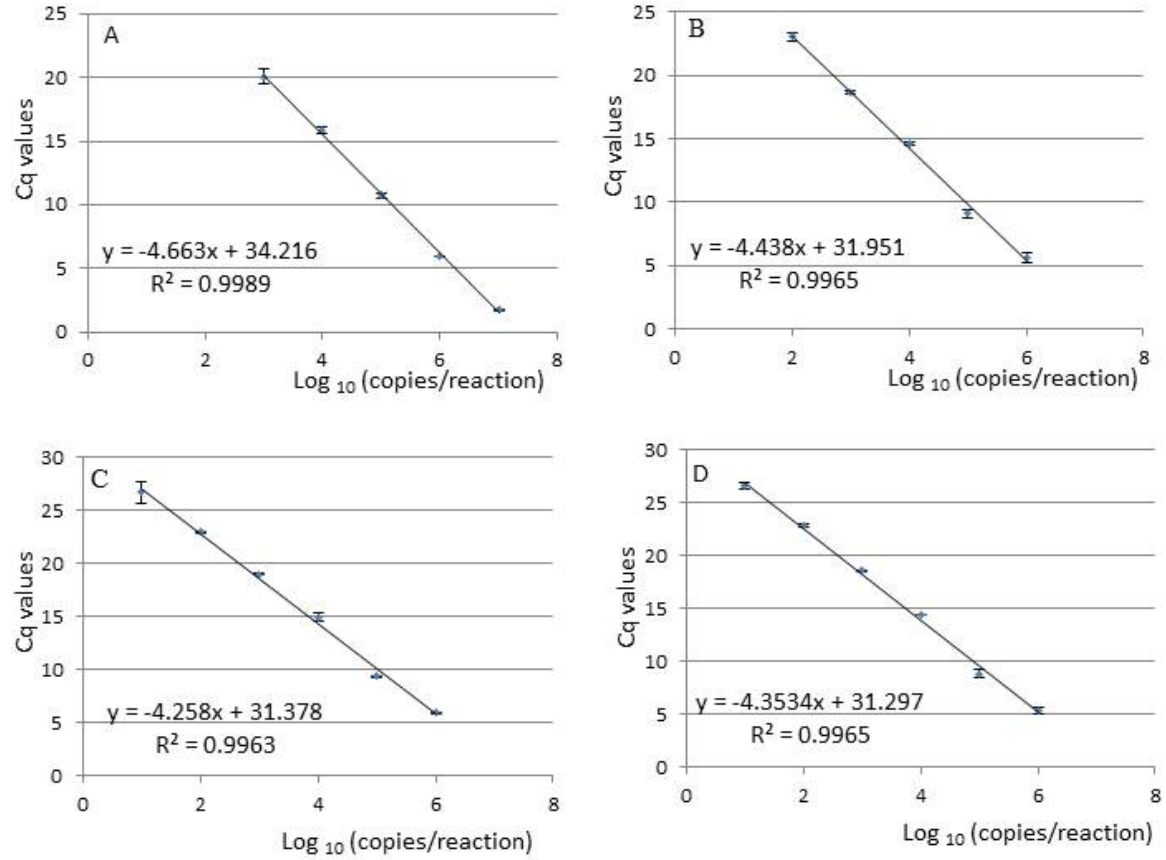

Figure 3. Standard curves for the norovirus GII real-time PCR assay using 10 -fold serial dilutions of the synthetic target. (A) Noprobe1. (B) Noprobe2. (C) Noprobe3. (D) Noprobe4. The assays were performed in duplicate, and the average values were plotted. Only the Cq values from the second round of PCR were used.

\section{Sequencing}

The PCR product of the norovirus samples was purified using the Wizard SV PCR Clean-Up System and ligated to a pCR4-TOPO vector (Thermo Fisher Scientific, Waltham, MA), according to the manufacturer's instruction. After transformation, 10 colonies for each PCR product were picked up and used for extraction of plasmids. Both strands of plasmids were sequenced using the Sanger sequencing method at the Uppsala Genome Center (Uppsala, Sweden).

\section{Results and discussion}

The sensitivity of our single-tube nested real-time PCR assay with long internally quenched probes for the detection of norovirus genogroup II was tested using a dilution series of $10^{6}$ to $10^{\circ}$ copies of a synthetic oligonucleotide. The Cq values from the second round of real-time PCR assays were used to plot standard curves (Figure 3). A linear relationship in the range 10-1,000,000 copies/reaction for the standard curves was observed when Noprobe3 and Noprobe4 were used in real-time PCR, with a detection limit of 10 copies/ reaction, though signals in 3 out of 6 wells calculated to contain a single copy were also detected. The detection limit for Noprobe1 was 1000 copies/reaction, while the detection limit for Noprobe2 was 100 copies/reaction.

The specificity of the real-time PCR assays was evaluated by testing 46

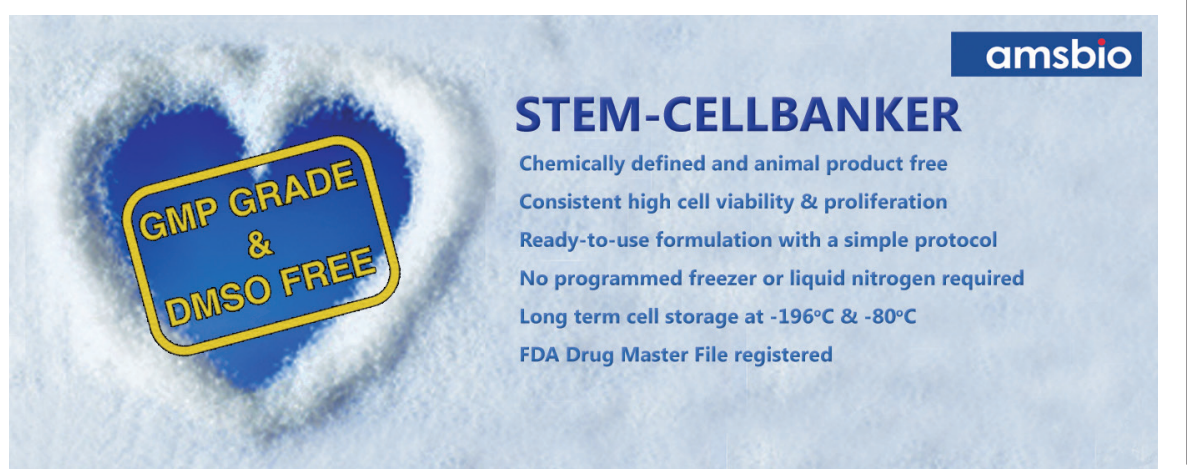

norovirus genogroup II samples, 23 norovirus genogroup I samples, and 20 samples testing negative for both norovirus genogroup I and genogroup II. In all assays, no cross-reactivity was observed for RNA from the 23 norovirus genogroup I samples and RNA from the 20 norovirus genogroup I and II negative samples. Among the 46 norovirus genogroup II samples, 44 samples were determined as norovirus genogroup II positive by all assays. The PCR products of the two remaining samples, which were false negatives compared with the routine PCR assays, were examined by agarose gel electrophoresis. A clear band with the expected size could be seen on the agarose gel (results not shown). This indicated that there might be a mismatch between the target and the probe. To identify possible sequence variation within the target region hybridized by the probe, the PCR products of these two samples were cloned into a pCR4-TOPO vector and sequenced. Long deletions within the target region hybridized by the probe were observed for both samples (results not shown). Sequencing results for 1 of the 2 samples indicated that it was a mixture of at least 2 virus variants with deletions of 51 and 39 nucleotides, respectively. We thus obtained an explanation for the absence of signal in these two samples: The probe could not bind to the target region due to long sequence deletions in the samples.

The PCR products of five other randomly selected norovirus Gll samples were also sequenced. The sequencing showed that the probe region can tolerate sequence variations of three different variants of the viruses (results not shown), which is in agreement with our previous study showing that long primers and probes can tolerate sequence variation (10). BLAST results using these five sequences as queries showed that the sequenced viruses belong to Genotype Gll.4, which was the most prevalent genotype in Northern Europe during the sampling period. A critical evaluation of the variation tolerance with a wider range of GIl norovirus samples remains to be done.

In the present work, all of the probes incorporated at least one internal quencher. The sensitivity of four different internally quenched probes (see Materials and methods) for the real-time detection of the amplification product of a 161 nucleotide sequence region of norovirus 
Gll was evaluated. We found that the triple-quenched probe Noprobe4 and the double-quenched probe Noprobe3 displayed the highest sensitivity. The sensitivity of Noprobe2 was less than that of Noprobe3 and Noprobe4, while Noprobe1 showed the lowest sensitivity. It was not possible to obtain information about the Zen structure for proprietary reasons. However, unlike other internal quenchers (here represented by NoProbe1 and NoProbe2), which may have a destabilizing effect when placed internally between nucleotides in a probe sequence, the Zen quencher is a non-base modifier that may actually strengthen duplex formation and thus does not reduce probe hybridization. Consequently, Zen can be placed close to the fluorophore, as in NoProbe3 and NoProbe4, contributing to efficient quenching. Thus, both low background and stabilization of the duplex contribute to the high sensitivity of the Zen quencher probes. Noprobe1 and Noprobe2 had the same internal BHQ1 quencher, but for Noprobe1 the BHQ was placed internally by BHQ DMT amidite chemistry (Figure 2). The introduction of the $\mathrm{BHQ}$ may create steric hindrance between neighboring nucleotides and between the target and the probe, which may affect annealing efficiency during PCR. For Noprobe2, the $\mathrm{BHQ}$ was placed internally by $\mathrm{BHQ} \mathrm{dT}$ linker amidite chemistry (Figure 2), through the introduction of a flexible linker between the quencher and the nucleotide. The linker may reduce the steric hindrance caused by the quencher, thereby improving the hybridization efficiency between the probe and its target at the annealing step. This probably explains the higher sensitivity of Noprobe2 compared with Noprobe1.

In comparison with traditional TaqMan real-time PCR assays for the detection of noroviruses, which use short primers and probes, here we have demonstrated that long primers and probes with an internal quencher can be applied in a TaqMan real-time PCR assay for the detection of highly variant norovirus Gll. The detection limit of the assays using Noprobe3 and Noprobe4 was similar to previous established assays at a range of 10-100 copies per reaction (16-17). With such high sensitivity, our assays cannot only be applied for clinical samples in which high copy numbers of viruses are present but also for detection of environmental and food samples where the virus is present at low copy numbers. This study shows that the VOCMA principle can be applied to real-time PCR for the quick and sensitive broad-range detection of highly variable pathogens. However, to ensure the high sensitivity of the real-time PCR assay, selection of appropriate internal quenchers is very important. This study paves the way for adaptation of our previous VOCMA system to multiplex real-time PCR assays, which are under development for the detection of pathogens of interest in several syndromic panels.

\section{Author contributions}

$H . X$. designed the study, performed experiments, analyzed data, and wrote the manuscript. S.G. optimized real-time PCR conditions and assisted with preparing the manuscript. C.Ö. provided all of the RNA samples in this study and assisted with preparing the manuscript. J.O. designed the study and assisted with preparing the manuscript. J.B. conceived the study and wrote the manuscript.

\section{Acknowledgments}

This work was supported by Aquavalens (Project No. 311846), within the European Union 7th framework programme, and by BALTINFECT (project No. 316275), within the European Union 7th framework programme (Sabine Gravelsina).

\section{Competing interests}

The authors declare no competing interests.

\section{References}

1. Scallan E., R.M. Hoekstra, F.J. Angulo, R.V. Tauxe, M.A. Widdowson, S.L. Roy, J.L. Jones, and P.M. Griffin. 2011. Foodborne illness acquired in the United States--major pathogens. Emerg. Infect. Dis. 17:7-15.

2. Trujillo A.A., K.A. McCaustland, D.P. Zheng, L.A. Hadley, G. Vaughn, S.M. Adams, T. Ando, R.I. Glass, and S.S. Monroe. 2006. Use of TaqMan real-time reverse transcription-

\section{Gene codes corporation \\ T C A C EN E \\ $A$ C T C O DES

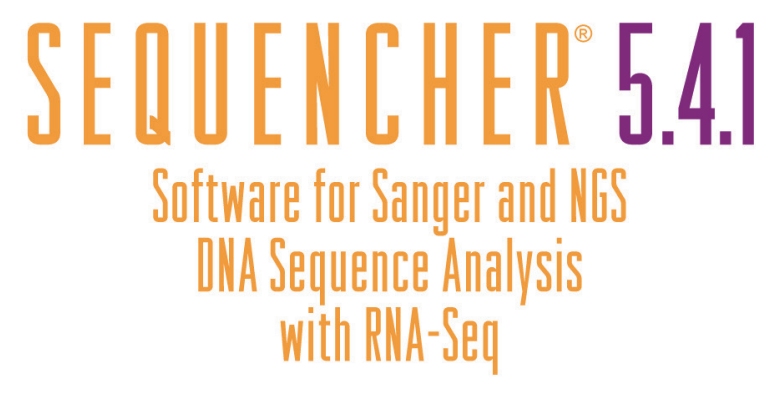

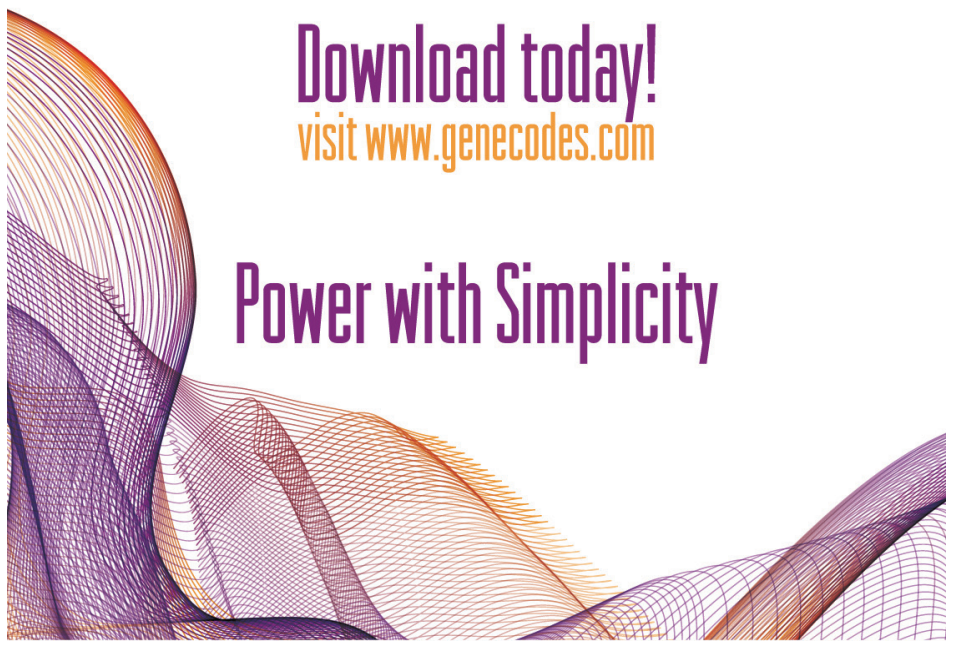


PCR for rapid detection, quantification, and typing of norovirus. J. Clin. Microbiol. 44:14051412.

3. Le Guyader, F.S., S. Parnaudeau, J. Schaeffer, A. Bosch, F. Loisy, M. Pommepuy, and R.L. Atmar. 2009. Detection and quantification of noroviruses in shellfish. Appl. Environ. Microbiol. 75:618624.

4. Kageyama, T., S. Kojima, M. Shinohara, K. Uchida, S. Fukushi, F.B. Hoshino, N. Takeda, and K. Katayama. 2003. Broadly reactive and highly sensitive assay for Norwalk-like viruses based on real-time quantitative reverse transcription-PCR. J. Clin. Microbiol. 41:15481557.

5. Henke-Gendo, C., G. Harste, B. JuergensSaathoff, F. Mattner, H. Deppe, and A. Heim. 2009. New real-time PCR detects prolonged norovirus excretion in highly immunosuppressed patients and children. J. Clin. Microbiol. 47:2855-2862.

6. Van Stelten, A., T.M. Kreman, N. Hall, and L.E. Desjardin. 2011. Optimization of a real-time RT-PCR assay reveals an increase of genogroup I norovirus in the clinical setting. J. Virol. Methods 175:80-84.

7. Mohamed, N., S. Belák, K.O. Hedlund, and J. Blomberg. 2006. Experience from the development of a diagnostic single tube real-time PCR for human caliciviruses, Norovirus genogroups I and II. J. Virol. Methods 132:69-76.

8. Pang, X.L., J.K. Preiksaitis, and B. Lee. 2005. Multiplex real time RT-PCR for the detection and quantitation of norovirus genogroups I and II in patients with acute gastroenteritis. J. Clin. Virol. 33:168-171.

9. Hymas, W., A. Atkinson, J. Stevenson, and D. Hillyard. 2007. Use of modified oligonucleotides to compensate for sequence polymorphisms in the real-time detection of norovirus. J. Virol. Methods 142:10-14.

10. Ohrmalm, C., R. Eriksson, M. Jobs, M. Simonson, M. Strømme, K. Bondeson, B. Herrmann, A. Melhus, et al. 2012. Variationtolerant capture and multiplex detection of nucleic acids: application to detection of microbes. J. Clin. Microbiol. 50:3208-3215.

11. Forsman, A., D. Uzameckis, L. Rönnblom, E. Baecklund, A. Aleskog, A. Bindra, R. Pipkorn, S. Lejniece, et al. 2003. Singletube nested quantitative PCR: a rational and sensitive technique for detection of retroviral DNA. Application to RERV-H/HRV-5 and confirmation of its rabbit origin. J. Virol. Methods 111:1-11.

12. Hu, Y. and I. Arsov. 2014. A rapid single-tube protocol for HAV detection by nested real-time PCR. Food Environ Virol. 6:189-195

13. Choi, Y., B.Y. Jeon, T.S. Shim, H. Jin, S.N Cho, and H. Lee. 2014. Development of a highly sensitive one-tube nested real-time PCR for detecting Mycobacterium tuberculosis. Diagn. Microbiol. Infect. Dis. 80:299-303.

14. Ohrmalm C., M. Jobs, R. Eriksson, S. Golbob, A. Elfaitouri, F. Benachenhou, M. Strømme, J. Blomberg. 2010. Hybridization properties of long nucleic acid probes for detection of variable target sequences, and development of a hybridization prediction algorithm. Nucleic Acids Res. 38:e195.
15. SantaLucia, J., Jr., H.T. Allawi, and P.A. Seneviratne. 1996. Improved nearest-neighbor parameters for predicting DNA duplex stability. Biochemistry 35:3555-3562.

16. Höhne, M. and E. Schreier. 2004. Detection and characterization of norovirus outbreaks in Germany: application of a one-tube RT-PCR using a fluorogenic real-time detection system. J. Med. Virol. 72:312-319.

17. Yan, Y., H.H. Wang, L. Gao, J.M. Ji, Z.J. Ge, X.Q. Zhu, P.Y. He, and Z.W. Chen. 2013. A one-step multiplex real-time RT-PCR assay for rapid and simultaneous detection of human norovirus genogroup I, II and IV. J. Virol. Methods 189:277-282.

Received 03 July 2015; accepted 16 September 2015.

Address correspondence to Hongyan Xia, SDepartment of Biomedical Sciences and Veterinary Public Health, Swedish University of Agricultural Sciences, Box 7028, 75007, Uppsala, Sweden. E-mail: Hongyan.Xia@slu.se

To purchase reprints of this article, contact: biotechniques@fosterprinting.com

\section{The New Gold Standard HTS Reader}

\section{PHERAstar ${ }^{\circledR}$ FSX}

\section{German engineering at its best.}

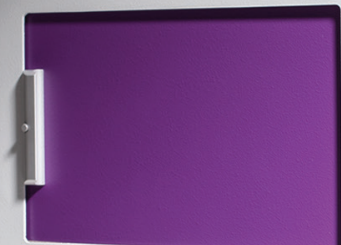

